\title{
The Importance of Screening Strongyloides stercoralis Infection in Solid Organ Transplant Donors and Recipients
}

\author{
Sara Abolghasemi $\mathbb{B}^{1}$, Farnaz Zolfaghari $\mathbb{i}^{2,{ }^{2}}$, Shahnaz Sali ${ }^{1}{ }^{1}$, Abdollah Ghaffari ${ }^{3}$ and Mehran Noori \\ (iD) 1 \\ ${ }^{1}$ Infectious Diseases and Tropical Medicine Research Center, Shahid Beheshti University of Medical Sciences, Tehran, Iran \\ ${ }^{2}$ Department of Infectious Diseases and Tropical Medicine, School of Medicine, Shahid Beheshti University of Medical Sciences, Tehran, Iran \\ ${ }^{3}$ Gholhak Laboratory, Tehran, Iran \\ "Corresponding author: Department of Infectious Diseases and Tropical Medicine, School of Medicine, Shahid Beheshti University of Medical Sciences, Tehran, Iran. Email: \\ zolfaghari83@yahoo.com
}

Received 2021 August 15; Revised 2021 November 13; Accepted 2021 November 14.

\begin{abstract}
Background: Strongyloides stercoralis is an intestinal nematode with worldwide distribution which is endemic in tropical and subtropical areas. It is unique in its ability to complete its entire life cycle and reinfect its human host via a phenomenon called autoinfection. The majority of infections are clinically asymptomatic or can manifest with gastrointestinal symptoms, such as heartburn, bloating, diarrhea, abdominal discomfort, gastroesophageal reflux disease (GERD), or symptoms related to the penetration of larvae through skin, including dermatitis and eosinophilia. Immunosuppressed patients, such as solid organ transplant patients with untreated strongyloidiasis are at great risk of hyperinfection, dissemination syndromes, and often fatal conditions associated with strongyloidiasis.

Methods: In this retrospective single-center cross-sectional study, we evaluated 157 cases (131 heart, lung, and kidney transplant recipients and 26 kidney donors) in Tehran, Iran, from March 2020 to April 2021. Demographic information including age, gender, place of birth, place of residence, underlying disease, being the donor or recipient, history of previous transplant and type of transplant, travel history to endemic regions, and seropositivity for $S$. stercoralis were evaluated.

Results: Among 157 cases with a mean age of 38.9 years, 40 (25.5\%) cases tested positive for S. stercoralis antibodies. Out of 26 donors, eight cases were seropositive, and among 131 recipients, 32 cases were seropositive. There was no significant correlation between birth place and place of residence in endemic areas and seropositivity for S. stercoralis. None of the cases showed hypereosinophilia, hyperinfection, or disseminated syndrome. Also, we found no significant correlation between the type and time of transplant, history of previous transplants, and S. stercoralis infection.

Conclusions: Universal screening for S. stercoralis latent infection is important in immunosuppressed patients, especially in solid organ transplants because of the high risk of hyperinfection and disseminated syndrome, which may culminate in death. Furthermore, screening for latent strongyloides infection in donors seems to be necessary because of the possibility of strongyloides infection being transmitted from donors to recipients.
\end{abstract}

Keywords: Strongyloides stercoralis, Solid Organ Transplant, Disseminated Syndrome, ELISA, Serology

\section{Background}

Strongyloides stercoralis is an intestinal nematode with worldwide distribution which is endemic in tropical and subtropical areas. Approximately 100 million people are infected, while billions are at risk of infection. S. stercoralis is unique in its ability to complete its entire life cycle and reinfect its human host, which is known as autoinfection (1).

The majority of infections are clinically asymptomatic or can manifest with gastrointestinal symptoms such as heartburn, bloating, diarrhea or abdominal discomfort, gastroesophageal reflux disease (GERD), or via the penetration of skin by larvae with dermatitis and eosinophilia. Strongyloides infection occurs through skin contact with contaminated soil (1).

Immunosuppressed patients such as solid organ transplant patients with untreated strongyloidiasis are at a great risk of hyperinfection and dissemination syndromes and often fatal conditions associated with strongyloidiasis. This process can lead to a high risk of death, with mortality estimates ranging from 35 to 100\% (1).

Therefore, early diagnosis and screening for S. sterco- 
ralis infection is of the utmost importance. Even transplant donors may be the source of infection for recipients, so most guidelines recommend pre-transplant screening with measurement of $S$. stercoralis antibody levels in recipients and donors from endemic areas. In Iran, the exact prevalence of this infection has not been recorded, and transplant centers do not act similarly to assess this infection (1).

\section{Objectives}

Using the enzyme-linked immunosorbent assay (ELISA) method, this study aimed to evaluate the prevalence of $S$. stercoralis infection in this high-risk group in Tehran, Iran, from March 2020 to April 2021.

\section{Methods}

In this retrospective single-center cross-sectional study, we evaluated the prevalence of S. stercoralis infection and seropositivity among 157 cases (131 heart, lung, and kidney transplant recipients and 26 kidney donors; 104 males vs. 53 females) in Tehran, Iran. Blood samples were collected from transplant donors and recipients from March 2020 to April 2021. Demographic information, including age, gender, place of birth, residence, underlying diseases, and gastrointestinal symptoms, being the donor or recipient, history of previous transplants and the type of transplant, and travel history to endemic regions were collected from hospital questionnaires and medical documents. Based on some recent literature, we considered humid coastal provinces in the north, south, and southwest of Iran as the endemic areas for S. stercoralis. All blood samples were centrifuged, and the serum was separated and analyzed for IgG antibodies using ELISA kits to detect $S$. stercoralis antibody. The method was carried out according to the instructions given by the manufacturers (Demeditec Company, Germany). The sensitivity and specificity of ELISA test were $87.9 \%$ and $95.8 \%$, respectively.

\section{Results}

We evaluated 157 cases, including 131 (83.5\%) heart, lung, and kidney recipients and 26 (16.5\%) kidney donors referred to our center for latent $S$. stercoralis screening. Among them, 40 cases (25.47\%) were seropositive. The mean age of patients was 38.9 years, and the age distribution of seropositivity is shown in Table 1 . In addition, 104 (66.2\%) subjects were males, and 53 (33.8\%) cases were females (Table 2).
We categorized the birth region and place of residence of all cases into the north, west, east, south, southeast, southwest, the center, and capital of Iran, and also assessed recent travel to endemic areas. We found no significant correlation between the type of transplant, history of previous transplants, the number of previous transplants, and the presence of comorbidities with seropositivity of S. stercoralis. Table 3 shows the frequency of seropositivity of $S$. stercoralis in transplant recipients and donors, and Table 4 depicts the comorbidities, type of transplant, and history of previous transplants.

We assessed the complete blood counts of all cases, but none of them showed hypereosinophilia and hyperinfection syndrome. Out of 15 immigrant cases, five were seropositive for $S$. stercoralis infection. Table 5 shows the birth region, residence place, immigration status, and recent travel to endemic regions. Gastrointestinal symptoms, such as diarrhea, abdominal discomfort, bloating, and heartburn were not detected in seropositive cases.

\section{Discussion}

Strongyloidiasis is a parasitic infection which involves persistent infection and dissemination, and in the most severe cases, the occurrence of potentially fatal disease. Despite the fact that there is no accurate estimation of its prevalence, this infection is spread throughout the world.

In our study, the seroepidemiological prevalence of $S$. stercoralis infection among heart, lung, and kidney transplant recipients and kidney donors from all provinces of Iran was $25.47 \%$ ( 40 cases from a total of 157 were seropositive).

Strongyloidiasis has been reported in various regions of Iran. Although the prevalence rates of this infection is unclear in some parts of the country, prior studies have demonstrated that it is still prevalent in the northern and southern parts of the country, which are considered as endemic areas. However, some experts believe that its prevalence in these regions is largely underestimated (2).

Rafiei et al. conducted a cross-sectional study to investigate the seroepidemiological prevalence of S. stercoralis infection among immunocompromised individuals in Ahvaz, southern Iran, in 2016. They assessed 270 immunocompromised patients consisting of 166 (61.5\%) human immunodeficiency virus (HIV) cases, 36 (13.3\%) leukemia cases, 68 (25.2\%) cancer cases, and 39 (14.4\%) immunocompromised individuals that were found to be positive. No statistically significant correlations were observed between age, gender, race, and the type of immunodeficiency and levels of S. stercoralis antibodies. The researchers concluded that there was a high prevalence of infection with 
Abolghasemi S et al.

\begin{tabular}{|c|c|c|c|c|}
\hline Age $(y)$ & Number & Frequency (\%) & \multicolumn{2}{|c|}{ Ig G Positive Number/Frequency (\%) } \\
\hline $\mathbf{0}-\mathbf{2 0}$ & 11 & 7.1 & 2 & 18.2 \\
\hline $21-40$ & 76 & 48.4 & 21 & 27.6 \\
\hline $41-60$ & 58 & 36.9 & 13 & 22.4 \\
\hline$>60$ & 12 & 7.6 & 4 & 33.3 \\
\hline Total & 157 & 100 & 40 & 25.47 \\
\hline Gender & Number & Frequency (\%) & \multicolumn{2}{|c|}{ Ig G Positive Number/Frequency (\%) } \\
\hline Male & 104 & 66.2 & 29 & 27.9 \\
\hline Female & 53 & 33.8 & 11 & 20.8 \\
\hline Total & 157 & 100 & 40 & 25.47 \\
\hline Donor/Recipients & Number & Frequency (\%) & \multicolumn{2}{|c|}{ Ig G Positive Number/Frequency (\%) } \\
\hline Lung recipients & 12 & 7.65 & 3 & 25 \\
\hline Heart recipients & 20 & 12.74 & 5 & 25 \\
\hline Kidney recipients & 99 & 63.05 & 24 & 24.2 \\
\hline Kidney donors & 26 & 16.56 & 8 & 30.7 \\
\hline Total & 157 & 100 & 40 & 25.47 \\
\hline
\end{tabular}

this particular parasite in immunocompromised individuals in southern Iran (2).

In our study, we found no significant correlation between places of birth or residence and seropositivity of $S$. stercoralis. This may be due to the recent increase in traveling that lead to the possibility of human to human transmission of the infection.

In our study, 15 cases were immigrants from Afghanistan and Iraq, among whom five tested positive for Strongyloides, which also indicates the importance of screening in immigrants.

In another study conducted by Al-Obaidi et al. in Houston, Texas, from 2012 to 2017, out of 1,689 kidney transplant candidates, 9.9\% were positive. All positive cases at the initial screening were administered ivermectin, and no cases of disseminated infection or hyperinfection were reported (3).

Winnicki et al. in Vienna University, Austria assessed the prevalence of $S$. stercoralis infection and hyperinfection syndrome in kidney transplant candidates over a 5year period. They reported that and six (3\%) patients were found to be positive, one of whom also experienced hyperinfection. None of the patients demonstrated hypereosinophilia, and eosinophil levels were below $5 \%$ in all cases $(4,5)$.
Meamar et al. investigated strongyloidiasis in 781 HIV+/AIDS patients using stool examinations, and two patients were found to be infected (6). Another study showed a 2.1\% S. stercoralis infection rate in the rural areas of Mazandaran province in Iran in 2013 (7). Other researchers have shown that $42 \%$ of the population with eosinophilia tested positive for $S$. stercoralis in Gilan province $(8,9)$, while another study conducted on mentally disabled individuals in southern Iran demonstrated that $17.3 \%$ of the residents were infected with this parasite (10).

In our study, $25 \%$ of cases tested positive, which was higher than some previous studies in Iran and other countries. Furthermore, 30\% of donors in our research were reported positive; therefore, screening for this particular parasite prior to transplantation is highly recommended in both organ donors and recipients.

Regarding the correlation between infection and gender, similar to other studies, there was no statistically significant difference, and the prevalence of infection in males and females was similar, with $27.9 \%$ of males and $20.8 \%$ of females tested positive for Strongyloides.

S. stercoralis transmission from a donor to recipient has been observed among solid organ transplant recipients. Hamilton et al. (11) reported that in two kidney transplant cases, the donor, who was born in the Dominican Repub- 


\begin{tabular}{|c|c|c|c|c|}
\hline & IgG Positive & Ig G Negative & Total & P-Value \\
\hline \multicolumn{5}{|l|}{ Number of patients } \\
\hline Male & 29 & 75 & 104 & 0.332 \\
\hline Female & 11 & 42 & 53 & \\
\hline Mean age & $38.9(5-73)$ & $38.98(4-72)$ & $38.94(4-73)$ & 0.975 \\
\hline Comorbidity & & & & 0.910 \\
\hline Hypertension & 2 & 5 & 7 & \\
\hline Rheumatologic disorders & 0 & 3 & 3 & \\
\hline Cirrhosis & 0 & 1 & 1 & \\
\hline Diabetes & 1 & 1 & 2 & \\
\hline Renal failure & 22 & 68 & 90 & \\
\hline Heart failure & 5 & 12 & 17 & \\
\hline Cystic fibrosis & 1 & 1 & 2 & \\
\hline Pulmonary failure & 2 & 8 & 10 & \\
\hline None & 7 & 18 & 25 & \\
\hline \multicolumn{5}{|l|}{ Type of transplant, No. (\%) } \\
\hline Kidney recipient & $24(24.2)$ & $75(75.8)$ & 99 & 0.998 \\
\hline Kidney donor & $8(30.8)$ & $18(69.2)$ & 26 & 0.498 \\
\hline Heart recipient & $5(25)$ & $15(75)$ & 20 & 0.998 \\
\hline Lung recipient & $3(25)$ & $9(75)$ & 12 & 0.998 \\
\hline History of previous transplant, No. (\%) & & & & 0.462 \\
\hline Yes & $7(31.8)$ & $15(68.2)$ & 22 & \\
\hline No & $33(24.4)$ & $102(75.6)$ & 135 & \\
\hline Order of the transplant, No. (\%) & & & & 0.837 \\
\hline First & $26(23.4)$ & $85(76.6)$ & 111 & \\
\hline Second & $6(33.3)$ & $12(66.7)$ & 18 & \\
\hline More than second & 0 & 2 & 2 & \\
\hline
\end{tabular}

lic, had been administered steroids before the organs were harvested. Researchers have also reported cases of donorderived infection in intestinal and liver transplant recipients whose donors were from endemic regions in Honduras and Ecuador $(12,13)$.

In another study involving targeted screening in donors with a history of residence in endemic areas conducted from 2010 to 2013 by the New York Organ Donor Network, it was observed that only 4.3\% (10 out of 233) of potential donors who had given consent had a positive ELISA for strongyloides against the crude antigen (14).

Hypereosinophilia, disseminated infection, and hyperinfection were not seen in any of our cases. Evaluation of $S$. stercoralis antibodies in transplant donors and recipients was actually a screening test for latent infection, and our patients were asymptomatic with no eosinophilia.
Detecting the larvae in stool samples can be quite challenging, with a minority of patients showing positive results, but serological testing (ELISA) for antibodies against S. stercoralis is an efficient means to detect the infection with a sensitivity and specificity of $88 \%$ and $94 \%$; thus, we used ELISA in our study. To the best of our knowledge, most of the prior studies carried out in Iran have utilized various stool examination methods, which lack the desired level of sensitivity, especially with regards to chronic and asymptomatic cases.

In a stool- and sero-survey for S. stercoralis performed in a community in the Peruvian Amazon region, the parasite was detected in the stools of $8.7 \%$ of individuals and also in the sera of $72 \%$ of the study population using ELISA. In a study carried out in Jamaica, Robinson et al. reported prevalence rates of $3.5 \%$ and $24.2 \%$ by stool examination 
Abolghasemi Set al.

\begin{tabular}{|c|c|c|c|c|}
\hline & IgG Positive & Ig G Negative & Total & P-Value \\
\hline Birth region in Iran & & & & 0.773 \\
\hline North & 6 & 10 & 16 & \\
\hline West & 4 & 10 & 14 & \\
\hline East & 2 & 9 & 11 & \\
\hline South & 2 & 6 & 8 & \\
\hline South east & 0 & 4 & 4 & \\
\hline South west & 6 & 18 & 24 & \\
\hline Center & 4 & 6 & 10 & \\
\hline Capital (Tehran) & 11 & 43 & 54 & \\
\hline Migrant & 5 & 11 & 16 & \\
\hline Place of residence & & & & 0.470 \\
\hline North & 5 & 9 & 14 & \\
\hline West & 4 & 6 & 10 & \\
\hline East & 0 & 4 & 4 & \\
\hline South & 4 & 6 & 10 & \\
\hline South east & 0 & 1 & 1 & \\
\hline South west & 5 & 10 & 15 & \\
\hline Center & 1 & 3 & 4 & \\
\hline Tehran (capital) & 21 & 78 & 99 & \\
\hline Recent travel to endemic region & & & & 0.447 \\
\hline Yes & 5 & 10 & 15 & \\
\hline No & 35 & 107 & 142 & \\
\hline Immigrant & & & & 0.534 \\
\hline Yes & 5 & 10 & 15 & \\
\hline No & 35 & 107 & 142 & \\
\hline
\end{tabular}

and ELISA, respectively. These findings confirm the higher sensitivity of serological tests compared to stool examinations. ELISA is widely considered as an excellent screening test for strongyloidiasis (15). Despite the high prevalence of this infection, there is limited knowledge concerning its epidemiology and seroepidemiology.

Al-Obaidi et al. in their study showed that a relatively significant number of cases (6.8\%) had seroconversion (negative to positive) during the study period, which indicates that serial serological screening may be needed if patients remain on the transplant waiting list for extended periods of time (3). There are also questions regarding the ideal frequency of screening and the possibility of exposures before or after transplantation. It is noteworthy that most patients had a history of travel to an endemic region. However, among 16 patients, three were born in the United States and had denied visiting an endemic region, which indicated a potential indigenous acquisition of $S$. stercoralis infection. Living donors were screened for strongyloides, but the screening was not done for deceased donors. A recent study performed in Miami, Florida, demonstrated a prevalence of 3.9\% in deceased donors. Thus, screening deceased donors should be carefully considered based on endemicity and the impact on the screening process $(16,17)$.

Universal screening tests have detected a large number of patients without risk factors, which indicates possible local acquisition of the infection. We would advocate serial screening when populations similar to ours are expected to wait long periods of time before transplantation. Although there is inadequate information regarding the prevalence of this disease in many parts of Iran, recent research indicates that $S$. stercoralis must be taken seriously. 


\subsection{Conclusions}

Due to the relatively high prevalence of S. stercoralis infection in Iran, universal screening for S. stercoralis latent infection is important in immunosuppressed patients, particularly for solid organ transplant candidates because of the high risk of hyperinfection and disseminated syndrome after transplantation, which may lead to death. Also, considering the fact that infection may be transmitted from donors to recipients, screening for latent strongyloides infection in donors seems to be necessary.

\section{Footnotes}

Authors' Contribution: S.A. conceived and designed the evaluation and drafted the manuscript. F.Z. participated in designing the evaluation, performed parts of the statistical analysis, and helped to draft the manuscript. Sh.S.reevaluated.

Conflict of Interests: We declare that we have no conflict of interest.

Data Reproducibility: The data presented in this study are openly available in one of the repositories or will be available on request from the corresponding author by this journal representative at any time during submission or after publication. Otherwise, all consequences of possible withdrawal or future retraction will be with the corresponding author.

\section{Ethical Approval: Ir.sbmu.msp.rec.1398.137s.}

Funding/Support: Infectious Diseases and Tropical Medicine Research Center, Shahid Beheshti University of Medical Sciences, Tehran, Iran funded this study.

\section{References}

1. Bennett JE, Dolin R, Blaser MJ. Mandell, Douglas, and Bennett's Principles and Practice of Infectious Diseases E-Book. 9th ed. Elsevier Health Sciences; 2019.

2. Rafiei R, Rafiei A, Rahdar M, Keikhaie B. Seroepidemiology of Strongyloides stercoralis amongst immunocompromised patients in Southwest Iran. Parasite Epidemiol Control. 2016;1(3):229-32. doi: 10.1016/j.parepi.2016.08.001. [PubMed: 29988178]. [PubMed Central: PMC5991854].

3. Al-Obaidi M, Hasbun R, Vigil KJ, Edwards AR, Chavez V, Hall DR, et al. Seroprevalence of Strongyloides stercoralis and Evaluation of Universal Screening in Kidney Transplant Candidates: A SingleCenter Experience in Houston (2012-2017). Open Forum Infect Dis. 2019;6(7). doi: 10.1093/ofid/ofz172. [PubMed: 31363770]. [PubMed Central: PMC6656655].
4. Miglioli-Galvao L, Pestana JOM, Santoro-Lopes G, Torres Goncalves R, Requiao Moura LR, Pacheco Silva A, et al. Severe Strongyloides stercoralis infection in kidney transplant recipients: A multicenter case-control study. PLoS Negl Trop Dis. 2020;14(1). e0007998. doi: 10.1371/journal.pntd.0007998. [PubMed: 32004346]. [PubMed Central: PMC7015428].

5. Winnicki W, Eder M, Mazal P, Mayer FJ, Sengolge G, Wagner L. Prevalence of Strongyloides stercoralis infection and hyperinfection syndrome among renal allograft recipients in Central Europe. Sci Rep. 2018;8(1):1-6. doi: 10.1038/s41598-018-33775-3. [PubMed: 30337607]. [PubMed Central: PMC6194009].

6. Meamar AR, Rezaian M, Mohraz M, Hadighi R, Kia EB. Strongyloides stercoralis hyper-infection syndrome in HIV+/AIDS patients in Iran. Parasitol Res. 2007;101(3):663-5. doi: 10.1007/s00436-007-0531-x. [PubMed: 17401580].

7. Ahmadi M, Beigom Kia E, Rezaeian M, Hosseini M, Kamranrashani B, Tarighi F. Prevalence of Strongyloides stercoralis and other intestinal parasites in rehabilitation centers in Mazandaran Province, northern Iran. J Mazandaran Univ Med Sci. 2015;25(130):1-7.

8. Fouladvand M, Barazesh A, Tahmasebi R. Seroepidemiological study of strongyloidiasis in patients taking immunosuppressive drugs in Bushehr, Iran, 2012. J Mazandaran Univ Med Sci. 2017;27(149):187-91.

9. Ashrafi K, Tahbaz A, Rahmati B. Strongyloides stercoralis: the most prevalent parasitic cause of eosinophilia in Gilan province, northern Iran. Iran J Parasitol. 2010;5(3):40.

10. Sharifdini M, Eshrat Beigom KIA, Ashrafi K, Hosseini M, Mirhendi H, Mohebali M, et al. An analysis of clinical characteristics of Strongyloides stercoralis in 70 indigenous patients in Iran. Iran J Parasitol. 2014;9(2):155.

11. Hamilton KW, Abt PL, Rosenbach MA, Bleicher MB, Levine MS, Mehta J, et al. Donor-derived Strongyloides stercoralis infections in renal transplant recipients. Transplantation. 2011;91(9):1019-24. doi: 10.1097/TP.ob013e3182115b7b. [PubMed: 21358367].

12. Rodriguez-Hernandez MJ, Ruiz-Perez-Pipaon M, Canas E, Bernal C, Gavilan F. Strongyloides stercoralis hyperinfection transmitted by liver allograft in a transplant recipient. Am J Transplant. 2009;9(11):2637-40. doi: 10.1111/j.1600-6143.2009.02828.x. [PubMed: 19843038].

13. Abanyie FA, Gray EB, Delli Carpini KW, Yanofsky A, McAuliffe I, Rana M, et al. Donor-derived Strongyloides stercoralis infection in solid organ transplant recipients in the United States, 2009-2013. Am J Transplant. 2015;15(5):1369-75. doi: 10.1111/ajt.13137. [PubMed: 25703251]. [PubMed Central: PMC4747246].

14. Levi ME, Kumar D, Green M, Ison MG, Kaul D, Michaels MG, et al. Considerations for screening live kidney donors for endemic infections: a viewpoint on the UNOS policy. Am J Transplant. 2014;14(5):1003-11. doi: 10.1111/ajt.12666. [PubMed: 24636427].

15. Robinson RD, Lindo JF, Neva FA, Gam AA, Vogel P, Terry SI, et al. Immunoepidemiologic studies of Strongyloides stercoralis and human T lymphotropic virus type I infections in Jamaica. J Infect Dis. 1994;169(3):692-6. doi: 10.1093/infdis/169.3.692. [PubMed: 8158055].

16. Hasan A, Le M, Pasko J, Ravin KA, Clauss H, Hasz R, et al. Transmission of Strongyloides stercoralis through transplantation of solid organs-Pennsylvania, 2012. Morb Mortal Wkly Rep. 2013;62(14):264.

17. Hoy WE, Roberts NJ, Bryson MF, Bowles C, Lee JC, Rivero AJ, et al. Transmission of strongyloidiasis by kidney transplant? Disseminated strongyloidiasis in both recipients of kidney allografts from a single cadaver donor. JAMA. 1981;246(17):1937-9. [PubMed: 7026811]. 